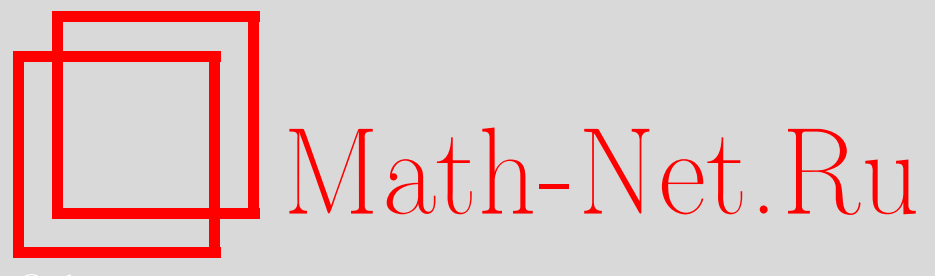

А. В. Чашкин, О реализации линейных булевых операторов неветвящимися программами с условной остановкой, Дискрет. матем., 1999, том 11, выпуск 1, 146-150

DOI: https://doi.org/10.4213/dm359

Использование Общероссийского математического портала Math-Net.Ru подразумевает, что вы прочитали и согласны с пользовательским соглашением http://www.mathnet.ru/rus/agreement

Параметры загрузки:

IP: 54.164 .48 .24

26 апреля 2023 г., 17:11:21 


\title{
О реализации линейных булевых операторов неветвящимися программами с условной остановкой
}

\author{
(c) 1999 г. А. В. Чашкин
}

\begin{abstract}
Рассматривается сложность реализации линейных булевых операторов неветвящимися программами с условной остановкой. Для почти всех таких операторов найдены точные по порядку оценки среднего времени вычисления их значений.

Работа выполнена при поддержке Российского фонда фундаментальных исследований, проект 96-01-01068, и ФЦП «Интеграция», проект 473.
\end{abstract}

В работе рассматривается сложность реализации линейных булевых операторов неветвящимися программами с условной остановкой. Неветвящиеся программы с условной остановкой отличаются от обычных неветвящихся программ тем, что некоторые из операторов и, возможно, некоторые из входов программы помечены целыми числами от 0 до $m$; операторы, помеченные нулем, останавливают выполнение программы, если их значение равно единице; $i$-м значением программы после ее остановки объявляется значение ее последнего выполненного оператора, помеченного числом $i$, а если такого оператора нет, то $i$-м значением программы объявляется значение входа, помеченного числом $i$.

Рассматриваемые программы на различных аргументах могут работать различное время, поэтому естественной мерой сложности таких программ является среднее время работы. Различные вопросы, связанные со средним временем вычисления значений булевых функций и булевых операторов неветвящимися программами с условной остановкой, ранее были рассмотрены в $[1,2]$. В этих работах можно найти все неопределяемые ниже понятия; касающиеся неветвящихся программ с условной остановкой. Полагаем, что число переменных всех рассматриваемых ниже операторов больше некоторой положительной постоянной.

Дадим необходимые определения.

Пусть $X=\left\{x_{1}, \ldots, x_{n}\right\}$ - множество независимых булевых переменных, переменной $x_{i}$ приписано некоторое число $a_{i}$ из множества $\{0,1, \ldots, m\}, i=1, \ldots, n$.

Неветвящейся программой с условной остановкой назовем последовательность $P=p_{1} \ldots p_{i} \ldots p_{s}$, элементами которой являются операторы $p_{i}=\left(f_{i}\left(p_{i, 1}, p_{i, 2}\right), a_{i}\right)$, где $a_{i} \in\{0,1, \ldots, m\}, f_{i}$ - двухместная булева функция, $p_{i, j} \in\left\{p_{1}, \ldots, p_{i-1}\right\} \cup X_{n}$. Оператор $p_{i}$ назовем оператором первого тига, или функциональным оператором, 
если $a_{i} \neq 0$, и оператором второго типа, или оператором остановки, если $a_{i}=0$. Будем говорить, что программа $P$ имеет $n$ входов, $m$ выходов, и ее $i$-у входу приписана переменная $x_{i}$.

Значение оператора $p$ программы $P$ на произвольном двоичном наборе $x$ определим индуктивно. Для первого оператора положим $p_{1}(x)=f_{1}(x)$, а при $i>1$ положим $p_{i}(x)=f_{i}\left(p_{i, 1}(x), p_{i, 2}(x)\right)$. Через $n(p)$ обозначим номер оператора $p$ в программе $P$, через $q_{t}-t$-й оператор второго типа этой программы. Пусть программа $P$ содержит $r$ операторов остановки. Через $q_{t, j}, t \in\{1,2, \ldots, r\}$, обозначим либо оператор $p_{i} \mathrm{c}$ наибольшим номером $i$, для которого $i<n\left(q_{t}\right)$ и $a_{i}=j$, либо, если такого оператора нет, переменную $x_{k}$ с максимальным индексом $k$, которой приписано число $j$. Результат действия программы $P$ на наборе $x$ обозначим через $P(x)=\left(P_{1}(x), \ldots, P_{m}(x)\right)$, значение его $j$-й компоненты определим посредством формулы

$$
\begin{aligned}
P_{j}(x)=q_{1}(x) q_{1, j}(x) \vee \bar{q}_{1}(x) & \left(q_{2}(x) q_{2, j}(x) \vee \ldots\right. \\
& \left.\vee \bar{q}_{r-2}(x)\left(q_{r-1}(x) q_{r-1, j}(x) \vee \bar{q}_{r-1}(x) q_{r}(x) q_{r, j}(x)\right) \ldots\right) .
\end{aligned}
$$

Временем работы $T_{P}(x)$ программы $P$ на наборе переменных $x$ назовем минимальное $n\left(q_{j}\right)$ такое, что $q_{j}(x)=1$. Без ограничения общности полагаем, что для каждого $x$ значение хотя бы одного оператора $q_{j}(x)$ отлично от нуля. Величину

$$
T(P)=2^{-n} \sum T_{P}(x)
$$

где суммирование производится по всем двоичным наборам длины $n$, назовем средним временем работы программы $P$. Если для некоторого булева оператора $f$ и любого двоичного набора $x$ справедливо равенство $f(x)=P(x)$, то будем говорить, что программа $P$ вычисляет оператор $f$. Величину

$$
T(f)=\min T(P)
$$

где минимум берется по всем программам, вычисляющим $f$, назовем средним временем вычисления оператора $f$. Программу $P$, вычисляющую оператор $f$, для которой справедливо равенство $T(f)=T(P)$, назовем минимальной программой. Число элементов минимальной схемы из функциональных элементов, реализующей оператор $f$ в базисе из всех двухместных функций, назовем сложностью $f$ и обозначим символом $L(f)$.

Легко видеть, что схемы из функциональных элементов являются частным случаем неветвящиеся программ с условной остановкой. Любую схему можно рассматривать как программу без операторов остановки. Поэтому очевидно, что

$$
T(f) \leqslant L(f) .
$$

Линейные булевы операторы, состоящие из $n$ функций и зависящие от $n$ переменных, далее будем называть линейными $(n, n)$-операторами.

Основным результатом работы является следующая теорема.

Теорема 1. Для каждого линейного $(n, n)$-оператора $f$ справедливо неравенство

$$
T(f) \leqslant \frac{n^{2}}{2 \log _{2} n}(1+o(1)) .
$$


Для почти всех линейных $(n, n)$-операторов $f$ справедливо неравенство

$$
T(f) \geqslant \frac{n^{2}}{6 \log _{2} n}(1+o(1)) .
$$

Доказательство. Верхняя оценка легко следует из существования вентильных схем, имеющих требуемую сложность [3]. Поэтому ограничимся доказательством нижней оценки.

Введем ряд необходимых понятий и установим некоторые свойства минимальных программ, реализующих линейные операторы.

Через $N(L, n)$ обозначим число неизоморфных программ в базисе из всех не более чем двухместных булевых функций, содержащих не более $L$ операторов, имеющих $n$ входов и $n$ выходов. Из леммы 4 в [2] следует, что

$$
N(L, n) \leqslant(c n L)^{2 L},
$$

где $c$ - некоторая постоянная.

Пусть $f$ - булев оператор, $P$ - программа, вычисляющая $f$. Каждому двоичному набору $x$ длины $n$, рассматриваемому как двоичная запись целого числа, поставим в соответствие его номер $N_{P}(x)$ такой, что $1 \leqslant N_{P}(x) \leqslant 2^{n} ; N_{P}(x)<N_{P}(y)$, если $T_{P}(x)<T_{P}(y) ; N_{P}(x)<N_{P}(y)$, если $T_{P}(x)=T_{P}(y)$ и $x<y$.

Пусть $H$ - произвольное подпространство в $\{0,1\}^{n}, d=\operatorname{dim} H$ и $\mathscr{B}=\left\{B_{i}\right\}-$ множество всех базисов в $H$. Рассматривая векторы из $H$ как целые числа, каждому базису $B_{i}=\left\{x_{1}^{i}, \ldots, x_{d}^{i}\right\}$ поставим в соответствие номер $-d$-разрядное число, $j$-й разряд которого есть число $x_{j}^{i}$. Базис с минимальным номером назовем минимальным базисом подпространства $H$.

Пусть $f-$ произвольный линейный оператор, $P$ - минимальная программа, вычисляющая $f$. Пусть $k$ - натуральный параметр, $x_{0}$ такое, что $N_{P}\left(x_{0}\right)=2^{n-k}+1$. Пусть $M-$ множество $x$ таких, что $N_{P}(x) \leqslant N_{P}\left(x_{0}\right), Y=\langle M\rangle$ - линейная оболочка множества $M, Z$ - дополнение к $Y$ в $\{0,1\}^{n}$, т.е. $Z$ - такое пространство, что $\{0,1\}^{n}=Y \otimes Z$ и $Y \perp Z$.

Заметим, что линейный оператор $f$ однозначно определяется первыми $T_{P}\left(x_{0}\right)$ операторами своей минимальной программы и множеством $\left\{f\left(z_{j}\right)\right\}$, где $\left\{z_{j}\right\}-$ минимальный базис в $Z$. Действительно, для однозначного определения линейного $(n, n)$-оператора достаточно знать его действие на $n$ линейно независимых вектоpax. Если известно $T_{P}\left(x_{0}\right)$ первых операторов, то можно найти множество $M$ и его образ $f(M)$, и, следовательно, образ базиса пространства $Y$. Таким образом, первые $T_{P}\left(x_{0}\right)$ операторов определяют действие $f$ на $\operatorname{dim} Y$ линейно независимых векторах. Значения оператора $f$ на оставшихся $n-\operatorname{dim} Y^{\prime}$ линейно независимых векторах явно зададим образом минимального базиса пространства $Z$. Так как множество $M$ состоит из $2^{n-k}+1$ векторов, то $\operatorname{dim} Y \geqslant n-k+1$. Следовательно,

$$
\operatorname{dim} Z \leqslant k-1
$$

т.е. базис пространства $Z$ содержит не более $k-1$ векторов.

Оценим число линейных операторов, для минимальных программ которых справедливо неравенство

$$
T_{P}\left(x_{0}\right) \leqslant \frac{n(n-k)}{6 \log _{2} n} .
$$


В силу неравенства (1) для числа $N$, равного числу различных программ, сложность которых не превосходит $T_{P}\left(x_{0}\right)$, справедливо неравенство

$$
N \leqslant\left(c n \frac{n(n-k)}{6 \log _{2} n}\right)^{2 n(n-k) /\left(6 \log _{2} n\right)} \leqslant 2^{n(n-k)} .
$$

Из последнего неравенства, неравенства (2) и сделанного выше замечания о величинах, определяющих линейные операторы, видим, что $R$ - число рассматриваемых операторов, не превосходит величины

$$
2^{n(n-k)} 2^{n(k-1)}=2^{n^{2}-n}=o\left(2^{n^{2}}\right) .
$$

Следовательно, все минимальные программы почти всех линейных $(n, n)$-операторов удовлетворяют условию: если $N_{P}\left(x_{0}\right)=2^{n-k}+1$, то

$$
\text { - } T_{P}\left(x_{0}\right)>\frac{n(n-k)}{6 \log _{2} n} \text {. }
$$

Положим $k=\left\lceil\log _{2} n\right\rceil$ и $W=\left\{x \mid N_{P}(x) \geqslant N_{P}\left(x_{0}\right)\right\}$. Тогда для среднего времени работы каждой программы $P$, удовлетворяющей $(3)$,

$$
\begin{aligned}
T(P) & =2^{-n} \sum_{x \in\{0,1\}^{n}} T_{P}(x)>2^{-n} \sum_{x \in W} T_{P}\left(x_{0}\right) \\
& \geqslant 2^{-n} T_{P}\left(x_{0}\right)|W|=2^{-n}\left(2^{n}-2^{n-k}\right) \frac{n(n-k)}{6 \log _{2} n} \\
& >\frac{n^{2}}{6 \log _{2} n}\left(1+O\left(n^{-1}\right)\right) .
\end{aligned}
$$

Теорема 1 допускает следующее обобщение.

Теорема 2. Для почти всех линейных $(n, n)$-операторов $f$ сложности

$$
L(f)=L \geqslant n
$$

справедливо равенство

$$
T(f)=\Theta(L(f)) .
$$

Доказательство теоремы 2 отличается от доказательства теоремы 1 выбором значения параметра $k$, в теореме 2 достаточно взять $k=1$, и использованием величины $c L$ с некоторой достаточно малой постоянной $c$ вместо величины $n(n-k) /\left(6 \log _{2} n\right)$ (см. (3)).

Введем функцию Шеннона $T(n)$, определенную стандартным образом:

$$
T(n)=\max T(f)
$$

где максимум берется по всем линейным булевым $(n, n)$-операторам. Из теоремы 1 очевидным образом вытекает следующее утверждение.

\section{Теорема 3.}

$$
\frac{n^{2}}{6 \log _{2} n}(1+o(1)) \leqslant T(n) \leqslant \frac{n^{2}}{2 \log _{2} n}(1+o(1))
$$


Пусть $\mathscr{P}_{t}-$ множество неветвящихся программ, каждая из которых содержит не более $t$ операторов остановки. Положим

$$
T_{t}(f)=\min T(P)
$$

где минимум берется по всем программам $P$, вычисляющим $f$ и таким, что $P \in \mathscr{P}_{t}$. Введем функцию

$$
T_{t}(n)=\max T_{t}(f),
$$

где максимум берется по всем линейным булевым $(n, n)$-операторам $f$.

Теорема 4. Пусть $t=o(n / \log n)$, тогда

$$
T_{t}(n)=\frac{n^{2}}{2 \log _{2} n}(1+o(1)) .
$$

Для почти всех линейных $(n, n)$-операторов $f$ справедливо неравенство

$$
T(f) \geqslant \frac{n^{2}}{2 \log _{2} n}(1+o(1)) .
$$

Доказательство теоремы 4 аналогично доказательству теоремы 1. Единственное отличие вносит тот факт, что для числа программ с ограниченным числом операторов остановки справедлива верхняя оценка $n^{t}(c L)^{L(1+o(1))}$.

В заключение отметим, что для многих естественных классов булевых функций (булевых операторов) нетрудно показать [1, 2], что использование даже небольшого (растущего) числа операторов остановки, позволяет для почти всех функций (операторов) сделать среднее время вычисления меньше соответствующего обычного времени вычисления. Теорема 4 показывает, что для класса линейных булевых операторов это не так.

\section{Список литературы}

1. Чашкин А. В. О среднем времени вычисления значений булевых функций. Дискретный анализ и исследование операций. (1997) 4, 60-78.

2. Чашкин А. В. О среднем времени вычисления булевых операторов. Дискретный анализ и исследование операций. (1998) 5, 88-103.

3. Нечипорук Э. И. О топологических принципах самокорректирования. Проблемъ кибернетики (1969) 21, 5-102.

Статья поступила 04.07.1998. 